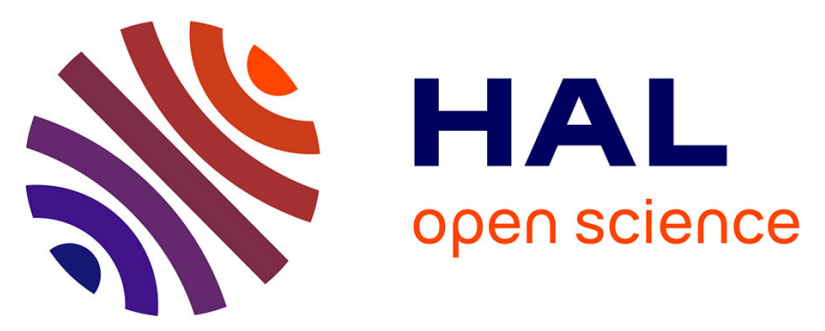

\title{
Discrete Digital Projections Correlation: a reconstruction-free method to quantify local kinematics in granular media by X-ray tomography
}

M.H. Khalili, Sébastien Brisard, Michel Bornert, Patrick Aimedieu, Jean-Michel Pereira, Jean-Noël Roux

\section{To cite this version:}

M.H. Khalili, Sébastien Brisard, Michel Bornert, Patrick Aimedieu, Jean-Michel Pereira, et al.. Discrete Digital Projections Correlation: a reconstruction-free method to quantify local kinematics in granular media by X-ray tomography. Experimental Mechanics, 2017, 57 (6), 10.1007/s11340-0170263-5 . hal-01474701

\section{HAL Id: hal-01474701 \\ https://hal-enpc.archives-ouvertes.fr/hal-01474701}

Submitted on 6 Mar 2017

HAL is a multi-disciplinary open access archive for the deposit and dissemination of scientific research documents, whether they are published or not. The documents may come from teaching and research institutions in France or abroad, or from public or private research centers.
L'archive ouverte pluridisciplinaire HAL, est destinée au dépôt et à la diffusion de documents scientifiques de niveau recherche, publiés ou non, émanant des établissements d'enseignement et de recherche français ou étrangers, des laboratoires publics ou privés. 
This is the post-print (ie final draft post-refereeing) version of the article Discrete Digital Projections Correlation: a reconstruction-free method to quantify local kinematics in granular media by X-ray tomography by M.H. Khalili, S. Brisard, M. Bornert, P. Aimedieu, J.-M. Pereira and J.-N. Roux. The final publication is available at Springer via https: //doi .org/10 . 1007/s11340-017-0263-5. 


\title{
Discrete Digital Projections Correlation: a reconstruction-free method to quantify local kinematics in granular media by $\mathrm{X}$-ray tomography
}

\author{
M.H. Khalili · S. Brisard • M. Bornert • P. Aimedieu • J.-M. Pereira · J.-N. Roux
}

the date of receipt and acceptance should be inserted later

\begin{abstract}
We propose a new method to measure the translations and rotations of each individual grain in a granular material imaged by computerized tomography. Unlike the classic approach, which requires that both initial and current configurations be fully reconstructed, ours only requires a reconstruction of the initial configuration. In this sense, our method is reconstruction-free, since any subsequent deformed state can be analyzed without further reconstruction. One distinguishing feature of the proposed method is that it requires very few projections of the deformed sample, thus allowing for time-resolved experiments.
\end{abstract}

Keywords Computed tomography · Digital image correlation · Full-field measurement - Granular material . Tomographic reconstruction

\section{Introduction}

The mechanical behaviour of granular materials has long been (and still is) investigated by means of macroscopic (e.g. oedometer and triaxial tests) experiments, see [1, 2, 3, 4]

M.H. Khalili · S. Brisard · M. Bornert · P. Aimedieu · J.-M. Pereira J.-N. Roux

Université Paris-Est, Laboratoire Navier (UMR 8205), CNRS, ENPC, IFSTTAR, Marne-la-Vallée F-77455, France

S. Brisard (corresponding author)

E-mail: sebastien.brisard@ifsttar.fr

M. Bornert

E-mail: michel.bornert@enpc.fr

P. Aimedieu

E-mail: patrick.aimedieu@enpc.fr

J.-M. Pereira

E-mail: jean-michel.pereira@enpc.fr

J.-N. Roux

E-mail: jean-noel.roux@ifsttar.fr among many others. Since the early eighties, computerized tomography [5, 6] has been successfully invoked to complement these global experiments, first to track collective events (e.g. the onset of shear bands [7, 8]), then to quantify the rigid-body motion of each individual grain [9, 10]. Together with numerical simulations based on the discrete element method (DEM) [11, 12, 13], these local measurements have the potential to deliver new insight on the complex behaviour of granular materials.

Full field measurements of 3D displacement fields are now performed almost routinely on 3D tomographic reconstructions by means of volumetric digital image correlation techniques. While the standard form of these techniques is better-suited to continua [14, 15, 16, 17, 18], discrete forms have later been devised with granular materials in mind (DVDIC [9], ID-Track [10]). They led to significant advances in the understanding of complex phenomena, such as strain localization. In particular, it was shown that grains undergo large rotations within shear bands [9] that may have a width of several grains. The importance of the overall angularity of the grains was also highlighted [10, 19].

Most volumetric digital image correlation techniques are based on the comparison of 3D images of the sample in its initial (undeformed) and current (deformed) states. Within the framework of computerized tomography, this means that a full tomographic scan is required in both initial and current states. This is a serious limitation for time-resolved experiments, as the total acquisition time of a full scan is of the order of the hour with laboratory facilities. While fast and ultra-fast tomography setups developed at synchrotron facilities [20, 21, 22] can overcome this limitation, such a solution is sometimes unpractical.

In this paper, an alternative route is explored in order to reduce the total acquisition time of the current configuration. Instead of reducing the acquisition time of each individual radiographic projection (as in fast and ultra-fast setups, rely- 
ing on very bright sources), we propose to reduce the overall number of projections itself. This idea is motivated by the fact that (assuming no breakage occurs) each grain undergoes a rigid body motion. Therefore, the current configuration is fully defined by a limited set of unknowns ( $3 N$ translational degrees of freedom and $3 N$ rotational degrees of freedom, where $N$ is the total number of grains), which suggests that a limited number of projections should suffice to accurately identify these unknowns and fully reconstruct the local displacements.

Owing to the insufficient number of projections, the downside of this approach is of course the impossibility of carrying out a 3D reconstruction of the sample in its current state, at least with standard methods making use of projections of the current configuration only. In other words, correlations cannot be performed on the 3D reconstructions, and we propose to skip the reconstruction step and directly match the radiographic projections instead. In this sense, our method can be considered as reconstruction-free, as it does not require the reconstruction of the current configuration. It should however be noted that together with the reconstruction of the reference configuration, the kinematics of each grain thus determined can be used to reconstruct a posteriori the current configuration, if needed.

The proposed method proceeds as follows. A full scan of the sample in its initial state is first carried out. A 3D image is reconstructed and each grain is segmented. The sample then undergoes a transformation, and a limited number of projections are acquired (target projections). Applying a trial rigid body motion to each grain (whose shape, local attenuation and initial position and orientation are known from the segmentation of the initial 3D reconstruction), the resulting trial projections can be computed. The trial rigid body motions are then optimized in order to minimize the discrepancy between the trial projections and the target projections.

Since correlations are evaluated on the radiographic projections, rather than the $3 \mathrm{D}$ reconstruction, our method is similar in spirit to the Projection-based Digital Volume Correlation (P-DVC) recently proposed by Leclerc, Roux and Hild [23, 24, 25, 26] in a continuous setting. Like the present work, P-DVC is based on a full reconstruction of the sample in its initial state, and a few projections of the sample in its current state. Unlike the present work, the displacement is assumed continuous and is interpolated between the nodes of a superimposed mesh and there is no need for segmentation of the initial state.

The method proposed here should therefore be understood as the discrete version of the work of Leclerc, Roux and Hild [24], just like DV-DIC [9] is the discrete version of V-DIC [14, 15]. For this reason, we will refer to our method as D-DPC, for Discrete Digital Projections Correlation.

It is clear from the above description of D-DPC that the proposed method can be seen as an inverse problem.
The corresponding forward problem consists in finding the radiographic projections of an assembly of grains subjected to trial rigid body motions. This forward problem is addressed in Sec. 2. It is formulated so as to minimize discretization errors, while allowing for an efficient implementation. Sec. 3 is devoted to the inverse problem itself. We first define the distance function which is used to measure the discrepancy between trial and target projections. A few synthetic test cases then illustrate the performance of the method. Finally, this paper closes in Sec. 4 with an application of the proposed method to true projections of gravel submitted to simple geometric transformations. An estimate of the measurement error is provided. Our method is shown to compare well with more conventional discrete correlation techniques, while requiring a substantially smaller acquisition time.

At this point, the terminology adopted throughout this paper ought to be clarified. First, the term "pixels" (denoted $p$ in this paper) will always refer to cells of the grid detector. Similarly, "voxels" (denoted $\underline{v}$ in this paper) will always refer to cells of the object space grid. The projection angle (rotation angle of the sample holder with respect to some reference direction) is denoted $\theta$. Finally, upper indices between round brackets refer to grains.

In general, bounds will be omitted in sums. Expressions like

$$
\sum_{\underline{p}} \ldots, \sum_{\underline{v}} \ldots, \sum_{\theta} \ldots \text { and } \sum_{i}(\ldots)^{(i)}
$$

should be understood as sums over all pixels, all voxels, all projections and all grains, respectively.

\section{The projection model}

The D-DPC method presented in this paper is formulated as an inverse problem. In view of solving this inverse problem, we first introduce a projection model which solves the following forward problem: find the radiographic projections of an assembly of objects (grains) which are subjected to trial rigid body motions. More precisely we assume that the geometry and initial position of the objects are known. Each object is subjected to an individual trial rigid body motion. In this section, we show how the projections of the resulting updated configuration can be computed by means of our projection model. In the remainder of this paper, objects which have been subjected to a rigid body motion will be called "transformed objects".

For untransformed objects, our model coincides with the approaches of most algebraic reconstruction techniques $[5,27]$. As such, it relies on the same assumptions. In particular, the in-plane dimensions of the pixels of the sensor are neglected. In other words, each pixel of the sensor is associated with a unique ray (no averaging over the surface 
of the pixel). For transformed objects, our model follows a Lagrangian approach: the rays are pulled back to the initial configuration. In the case of voxel-based geometrical descriptions of the objects, this reduces the accuracy losses.

The projection model is first developed in Sec. 2.1 for a single object subjected to a rigid body motion. It is then extended in Sec. 2.2 to an assembly of objects (e.g. granular materials), each object being subjected to an individual rigid body motion. Sec. 2.3 finally addresses some implementation issues for voxel-based representations of the grains.

2.1 Projections of a single object subjected to a rigid body motion

In the present paper, we consider a general tomography setup. The sample $\mathcal{B}$ is placed on a rotating stage. $\Delta$ denotes its axis of rotation; it is oriented by the unit vector $\underline{e}_{A}$. The origin $O$ is placed on the rotation axis $\Delta$, so that points can be identified in the remainder of this paper with their radius vector.

The sample is illuminated by an X-ray source (parallel or cone-beam), and the resulting projection is measured on a plane detector $\mathcal{D}$. Each point $p \in \mathcal{D}$ of the detector is hit by a unique $\mathrm{X}$-ray, the direction of which is given by the unit vector $\underline{T}(p)$, oriented from the source to the detector. We will assume that pixel values returned by the detector correspond to the intensity at the center of the pixel; therefore, $p \in \mathcal{D}$ will usually refer in the present paper to the center of a pixel of the detector. For parallel projections, we have $\underline{T}(p)=$ const. (see Fig. 1), while for cone-beam projections (see Fig. 2)

$\underline{T}(\underline{p})=\frac{\underline{p}-\underline{a}}{\|\underline{p}-\underline{a}\|}$,

where $\underline{a}$ denotes the location of the X-ray point source (apex of the cone); in the case of parallel tomography, $\underline{a}$ is located at infinity.

No assumption is made regarding the geometry of the setup. In particular, it is not assumed that the detector $\mathcal{D}$ is parallel to the axis of rotation $\Delta$ of the sample stage. Likewise, it is not assumed that the plane which contains the axis of rotation $\Delta$ and the point source $a$ is perpendicular to the detector $\mathcal{D}$. Indeed, our method is formulated in an intrinsic way which does not require perfect geometries.

For the sake of simplicity, it will however be assumed in most applications presented below that the geometry of the setup is indeed "perfect": for parallel setups, the detector and the axis of rotation of the sample are parallel and $\underline{T}(p)$ is normal to the detector, while for cone-beam setups, the axis of rotation of the sample is parallel to the detector, and the plane formed by the point source and the axis of rotation is perpendicular to the detector.

In both cases of perfect geometries, it is convenient to introduce a global frame $\left(O, \underline{e}_{x}, \underline{e}_{y}, \underline{e}_{z}\right)$ defined as follows (see

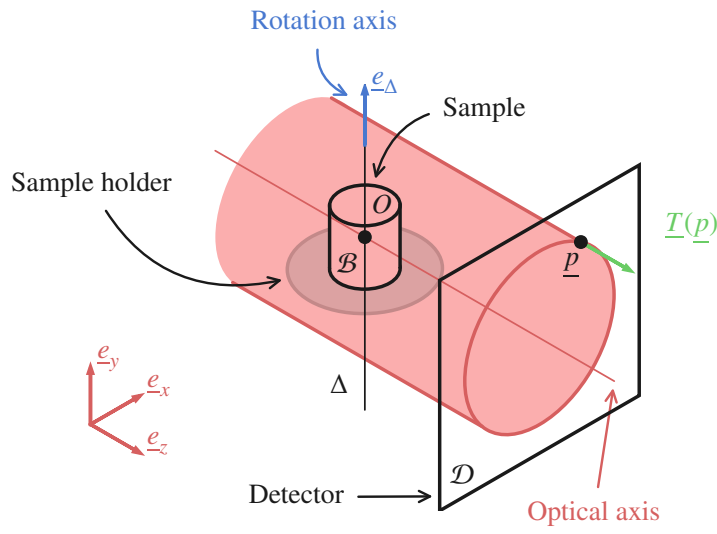

Fig. 1 Tomography setup for parallel (synchrotron) tomography.

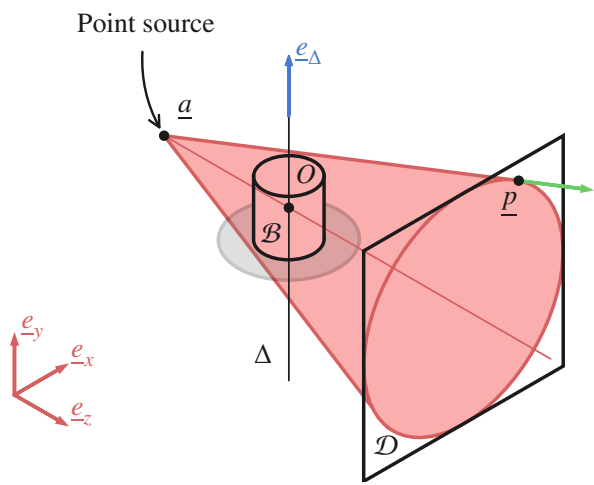

Fig. 2 Tomography setup for cone-beam (laboratory) tomography.

also Figs. 1 and 2). The unit vector $\underline{e}_{z}$, normal to the detector, points in the direction of propagation of the X-rays. $\underline{e}_{y}=\underline{e}_{A}$ is the (ascending) direction of the axis of rotation. Finally, $\underline{e}_{x}=\underline{e}_{y} \times \underline{e}_{z}$. Besides, for cone-beam setups, the origin $O$ is placed at the intersection between the normal to the detector passing through the point source, and the axis of rotation.

A body $\mathcal{B}$ is placed on the sample holder. The map $\underline{x} \in$ $\mathcal{B} \mapsto \mu(\underline{x})$ denotes its local linear absorption coefficient. Then, from the Beer-Lambert law, we get the following projection formula

$\ln \left[I_{0} / I\right](\underline{p})=\int \mu(\underline{p}+s \underline{T}(\underline{p})) \mathrm{d} s$,

where $s$ denotes the arc-length along the ray $(p, \underline{T}(p)), I_{0}$ and $I$ are the incident and transmitted intensities. In practice, for each pixel of the sensor, the ratio $\left[I_{0} / I\right](p)$ is deduced from the grey level. This preliminary calibration corrects for the possible non uniformity of the incident intensity and of the sensitivity of the individual pixels of the sensor [28]. 
It might be argued that the Beer-Lambert law (2) does not fully account for the complex physical phenomena governing the formation of a radiographic projection. It is assumed that $(i)$ phase contrast is negligible in front of attenuation contrast [29] and (ii) the X-ray source is monochromatic and beam hardening effects are disregarded [30]. Most tomographic reconstruction techniques currently in use rely on these assumptions; experience shows that these methods perform extremely satisfactorily, even on scans acquired in a laboratory (polychromatic) setup. Our method is nothing but a constrained classical algebraic reconstruction technique. As such, the use of the Beer-Lambert law (2) is not more (but not less) questionable for our method than for traditional reconstruction techniques.

$\mathcal{B}$ is now submitted to a rigid body motion prior to projection. This motion is defined by the translation vector $\underline{u} \in \mathbb{R}^{3}$, the rotation center $\underline{c}$ and the rotation tensor $\underline{\Omega} \in \mathrm{SO}(3)$, so that the point initially located at $\underline{X}$ is transported to $\underline{x}$

$\underline{x}=\underline{\underline{\Omega}} \cdot(\underline{X}-\underline{c})+\underline{u}+\underline{c}$.

The above equation is intrinsic in the sense that it does not require a frame of reference; as such, $\underline{\Omega}$ should really be understood as a rotation tensor. In the $\overline{\overline{g l}}$ obal frame of reference $\left(O, \underline{e}_{x}, \underline{e}_{y}, \underline{e}_{z}\right)$ introduced above, this rotation tensor can be represented by a rotation matrix.

Observing that the absorption coefficient $\mu$ is conserved during the motion, the projection at pixel $p$ of the transformed sample results from the combination of $\bar{E} q s$. (2) and (3)

$\ln \left[I_{0} / I\right](\underline{p})=\int \mu\left(\underline{\underline{\Omega}}^{T} \cdot[\underline{p}+s \underline{T}(\underline{p})-\underline{u}-\underline{c}]+\underline{c}\right) \mathrm{d} s$.

In a tomography experiment, the sample stage is rotated by an angle $\theta$ about the axis of rotation $\Delta$, oriented by the unit vector $\underline{e}_{\Delta}$ (see Figs. 1 and 2 ); $\underline{R}_{\theta}$ denotes the corresponding rotation tensor (the rotation center is the origin).

Composing the rigid body motion of the body and the rotation of the sample stage, it is readily seen that the point initially located at $\underline{X}$ is transported to $\underline{x}$, given by

$\underline{x}=\underline{R}_{\theta} \cdot \underline{\underline{\Omega}} \cdot(\underline{X}-\underline{c})+\underline{R}_{\theta} \cdot(\underline{u}+\underline{c})$,

while the final expression of the projection at pixel $\underline{p}$ and angle $\theta$ of the transformed body $\mathcal{B}$ reads

$\ln \left[I_{0} / I\right](\underline{p}, \theta)=\int \mu\left(\underline{\underline{\Omega}}^{T} \cdot\left[\underline{R}_{\theta}^{T} \cdot(\underline{p}+s \underline{T}(\underline{p}))-\underline{u}-\underline{c}\right]+\underline{c}\right) \mathrm{d} s$.

\subsection{Extension of the projection model to granular materials}

We now consider a granular medium. It is submitted to a mechanical loading, causing each grain to undergo a rigid body motion. Prior to loading, a first set of projections leads to a reconstruction of the 3D map $\underline{x} \mapsto \mu(\underline{x})$ of the linear attenuation in the reference configuration (unloaded sample, unrotated sample stage).

The linear absorption coefficient of air is significantly smaller than that of the grains and will be neglected in the remainder of this paper; in other words, it is assumed that $\mu(\underline{x})=0$ outside the grains.

The reconstructed image is then segmented; in other words, the total attenuation $\mu$ is decomposed as follows

$\mu(\underline{x})=\sum_{i} \mu^{(i)}(\underline{x})$

where the sum extends to all grains, and $\mu^{(i)}$ denotes the local attenuation of the $i$-th grain $\left(\mu^{(i)}(\underline{x})=0\right.$ outside grain $\left.i\right)$.

The rigid body motion of grain $i$ is defined by the translation vector $\underline{u}^{(i)}$, the rotation center $\underline{c}^{(i)}$ and the rotation tensor $\underline{\underline{\Omega}}^{(i)}$. The projection of this grain is then retrieved from Eq. (6)

$$
\begin{aligned}
\hat{P}^{(i)}\left(\theta, \underline{p} ; \underline{u}^{(i)}, \underline{\Omega}^{(i)}\right)= & \int \mu^{(i)}\left(\underline { \underline { \Omega } } ^ { ( i ) } { } ^ { T } \cdot \left[\underline{R}_{\theta}^{T} \cdot(\underline{p}+s \underline{T}(\underline{p}))\right.\right. \\
& \left.\left.-\underline{u}^{(i)}-\underline{c}^{(i)}\right]+\underline{c}^{(i)}\right) \mathrm{d} s .
\end{aligned}
$$

It should be noted that arbitrary choices of the rotation centers $\underline{c}^{(i)}$ (which are licit) might induce artificially large variations of the amplitude of the translation $\underline{u}^{(i)}$ from grain to grain, which in turn might lead to convergence issues for the inverse problem considered in Sec. 3. To avoid such issues, the center of rotation $\underline{c}^{(i)}$ of grain $(i)$ was placed at its center of mass. In other words, $\underline{u}^{(i)}$ is the translation of the center of mass of grain $i$.

Summing Eq. (8) over all grains, we get the following expression of the projection of the deformed sample

$\hat{P}\left(\theta, \underline{p} ; \underline{u}^{(1)}, \underline{\underline{\Omega}}^{(1)}, \ldots, \underline{u}^{(n)}, \underline{\underline{\Omega}}^{(n)}\right)=\sum_{i} \hat{P}^{(i)}\left(\theta, \underline{p} ; \underline{u}^{(i)}, \underline{\underline{\Omega}}^{(i)}\right)$.

The above expression defines our projection model, which solves the forward problem. In other words, if the translations and rotations of each grain are known, we can evaluate the projections of the deformed granular material.

For later use in the inverse problem (17) which defines the D-DPC method, the grain rotations $\underline{\Omega}^{(i)}$ must be parameterized. We use Rodrigues' formula [ $[\overline{\overline{3}}, 32]$ to map the rotation vector $\underline{\omega}=\omega \underline{n}$ ( $\omega$ : angle of rotation; $\underline{n}$ : axis of rotation, $\|\underline{n}\|=1$ ) to the rotation tensor $\underline{\underline{\Omega}}$

$\underline{\underline{\Omega}}=\underline{\underline{I}}+\frac{\sin \omega}{\omega} \underline{\underline{\omega}}+\frac{1-\cos \omega}{\omega^{2}} \underline{\underline{\omega}}^{2}=\exp \underline{\underline{\omega}}$,

where we have introduced the skew-symmetric tensor $\underline{\underline{\omega}}$ such that $\underline{\underline{\omega}} \cdot \underline{x}=\underline{\omega} \times \underline{x}$ for all $\underline{x} \in \mathbb{R}^{3}$. It should be noted that this parameterization is not differentiable at some points (namely, $\omega=2 \pi$ ) [33,34], which might cause issues with gradient-based optimization algorithms. In the present study, 
the rotations are relatively small, so that it was not required to consider this corner case.

We then introduce the compact notation

$$
\begin{aligned}
\mathbf{q}=\left[u_{1}^{(1)}, u_{2}^{(1)}, u_{3}^{(1)}, \omega_{1}^{(1)}, \omega_{2}^{(1)}, \omega_{3}^{(1)}, \ldots\right. \\
\left.\ldots, u_{1}^{(n)}, u_{2}^{(n)}, u_{3}^{(n)}, \omega_{1}^{(n)}, \omega_{2}^{(n)}, \omega_{3}^{(n)}\right]^{\top},
\end{aligned}
$$

which gathers in a unique column-vector the parameters defining the rigid body motion of each grain. The projection model defined by Eqs. (8) and (9) can then be written

$\hat{P}(\theta, \underline{p} ; \mathbf{q})=\sum_{i} \hat{P}^{(i)}\left(\theta, \underline{p} ; \mathbf{q}^{(i)}\right)$

where $\mathbf{q}^{(i)}=\mathbf{S}^{(i)} \cdot \mathbf{q}$ and the $6 n \times 6$ matrix $\mathbf{S}^{(i)}$ selects in $\mathbf{q}$ the rows corresponding to $\underline{u}^{(i)}$ and $\underline{\omega}^{(i)}$

$\mathbf{S}^{(i)}=[\underbrace{\mathbf{O}_{6}, \ldots, \mathbf{O}_{6}}_{i-1 \text { times }}, \mathbf{I}_{6}, \underbrace{\mathbf{O}_{6}, \ldots, \mathbf{O}_{6}}_{n-i \text { times }}]$,

( $\mathbf{O}_{6}: 6 \times 6$ null matrix; $\mathbf{I}_{6}: 6 \times 6$ identity matrix).

\subsection{Implementation of the projection model}

In situations of practical interest, the 3D map of the linear attenuation results from an initial 3D reconstruction of the (undeformed) granular sample. It is therefore voxelized, and we write the attenuation as the following discrete sum

$\mu(\underline{x})=\sum_{\underline{v}} \mu(\underline{v}) \chi(\underline{x}-\underline{v})$,

where $\underline{v}$ denotes the center of the current voxel and $\chi$ is the indicator function of the voxel centered at the origin. The projection formula (2) then becomes

$\ln \frac{I_{0}}{I(\underline{p})}=\sum_{\underline{v}} \mu(\underline{v}) \int \chi(\underline{p}-\underline{v}+s \underline{T}(\underline{p})) \mathrm{d} s$,

where the integral in the above equation is the chord length of the voxel centered at $\underline{v}$ intersected by the ray $(p, \underline{T}(p))$. Thus, the value of the projection is the summation of the chord lengths weighted by their attenuation coefficients $\mu(\underline{v})$. This summation is referred to as the radiological path and can be efficiently evaluated by means of Siddon's algorithm [35]. The improved version proposed by Jacobs [36] was implemented here.

Eq. (15) should be evaluated for every pixel of the detector. In Siddon's algorithm, unnecessary computations are avoided by considering only the voxels that are actually intersected by the current ray. A further gain can be made by only considering the rays that intersect the grain: to this end, bounding boxes are attached to each grain.

To close this section, we note that our projection model is based on a Lagrangian approach. In order to compute the projection of a transformed object, the inverse transform is applied to the rays, and the untransformed object is then projected along these Lagrangian rays. The alternative, Eulerian approach might seem more natural: the direct transform is applied to the object itself, which is then projected. However, this latter approach would require to discretize the (already discretized) object over a rotated grid. Our Lagrangian approach avoids the additional data losses that might possibly result from this rediscretization.

\section{The Discrete Digital Projection Correlation method}

The projection model presented in Sec. 2 allows to generate digital projections of an assembly of grains subjected to arbitrary rigid body motions. It is recalled that $\hat{P}(\theta, p ; \mathbf{q})$ denotes the resulting digital projection $(\theta$ : rotation angle of the sample holder; $p$ : center of detector pixel; q: generalized displacements of grains). We are now in a position to provide a definition of the Discrete Digital Projection Correlation method (D-DPC), which is formulated as an inverse problem, where the above projection model is used as the forward solver.

\subsection{Formulation of the method}

We consider a granular sample undergoing a geometric transformation resulting from e.g. mechanical loading. As already argued in Sec. 2.2, it is assumed that a full set of projections of the initial configuration is available, allowing for a fine description of the geometry and position of each grain. We then record a few projections $P(\theta, p)$ of the sample in its deformed (current) state. For each trial generalized displacement $\mathbf{q}$ of the grains, we compute the corresponding set of trial projections $\hat{P}(\theta, p ; \mathbf{q})$, and evaluate the discrepancy between experimental and trial projections. Minimizing this discrepancy with respect to $\mathbf{q}$ then leads to an estimate of the displacements of each grain. In the present work, we selected the following objective function as a measure of the discrepancy between $P$ and $\hat{P}$

$\mathrm{F}(\mathbf{q})=\sum_{\theta} \sum_{\underline{p}}(\hat{P}(\theta, \underline{p} ; \mathbf{q})-P(\theta, \underline{p}))^{2}$,

where the sum runs over the limited set of projection angles $\theta$ and all pixels $p$ of the detector. Minimization of the objective function $\mathrm{F}$ is known to deliver the maximum likelihood estimate of $\mathbf{q}$ for projections corrupted with Gaussian noise. In the more realistic case of Poisson noise, a different cost function ought to be adopted [37]; this is ongoing work.

To sum up, the grain displacements are retrieved from the following optimization problem

$\mathbf{q}^{\star}=\underset{\mathbf{q}}{\arg \min } \mathrm{F}(\mathbf{q})$. 
Numerical optimization of the cost function is carried out with the Levenberg-Marquardt method, which is wellsuited to nonlinear least-squares problems. At this stage, its performance has not been compared with other optimization techniques. It requires the partial derivatives of $\hat{P}$ with respect to the parameters $\mathbf{q}$, which are estimated by finite differences. Our implementation accounts for the sparsity of the resulting Jacobian matrix [see Eq. (12)]: the partial derivatives of $\hat{P}^{(i)}$ with respect to $\mathbf{q}^{(j)}$ are not evaluated for $j \neq i$. Furthermore, $\hat{P}^{(i)}$ and its derivatives are evaluated simultaneously to avoid redundant function calls. Nonlinear optimization methods are known to be sensitive to the initial guess; this point will be addressed in Sec. 3.3.

\subsection{Validation of the method}

In this section, we present a few test-cases of the D-DPC method, where the "experimental" projections $P(\theta, p)$ [see Eq. (16)] are in fact generated numerically from reference images of grains by means of our projection model. In other words,

$P(\theta, \underline{p})=\hat{P}\left(\theta, \underline{p} ; \mathbf{q}_{\text {exact }}\right)$

where $\mathbf{q}_{\text {exact }}$ is the generalized displacement that we impose to the grains and expect to retrieve through minimization of the cost function F [see Eq. (16)].

The simulations presented here are restricted to the case of parallel projection of two-dimensional objects in the $x z$ plane (perfect geometry, see Fig. 1). The rigid body motions of the grains are then characterized by three scalars (two displacements and one angle of rotation): let $u^{(i)}$ and $w^{(i)}$ denote the translation of the center of mass of grain $i$ along the $x$ and $z$ directions, and $\omega^{(i)}$ its angle of rotation about its center $\left(x^{(i)}, z^{(i)}\right)$.

It should be observed that displacements along the $\underline{\underline{R}}_{\theta}^{T}$. $\underline{e}_{z}$ direction induce no change in the projection at angle $\theta$. Hence, at least two projections are needed to fully resolve the displacement of a grain.

The simulations presented below are successful if $\mathbf{q}_{\mathrm{D}-\mathrm{DPC}}=$ $\mathbf{q}_{\text {exact }}$ (up to a specified tolerance), where $\mathbf{q}_{\text {D-DPC }}$ denotes the D-DPC estimate of the displacements. The initial guess for the optimization algorithm will always be taken as the reference state $\left(\mathbf{q}_{\text {init }}=\mathbf{0}\right)$

Fig. 3 shows a digital image of the grains considered here. This image corresponds to the reference (initial) configuration, $\mathbf{q}=\mathbf{0}$. In order to ensure that we explored realistic shapes and sizes of grains, as well as gray level variations within grains, this image was extracted from the (experimental) tomographic reconstruction of a real granular material. However, it is again emphasized that the projections $P(\theta, p)$ are generated numerically from this experimental image.

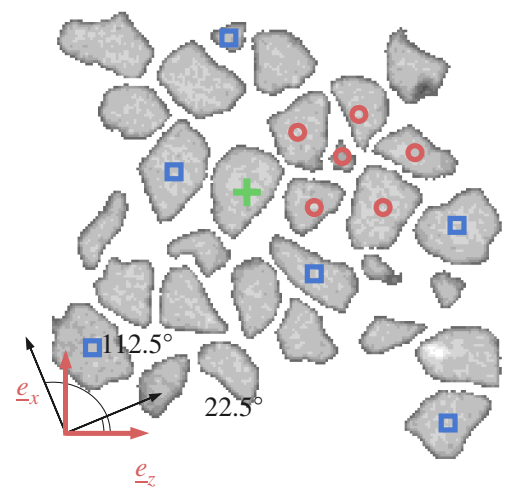

Fig. 3 Image of the 30 grains considered for the validation of the DDPC method in Sec. 3.2. The blue squares (resp. red circles) indicate the grains belonging to the "loose" (resp. "dense") set. The typical diameter of the grains is about 30 pix.

\subsubsection{Validation with two projections and small displacements}

In this section, only two projections are considered, at angles $\theta=22.5^{\circ}$ and $112.5^{\circ}$ (see Fig. 3). All components of the applied rigid body motion $\mathbf{q}_{\text {exact }}$ are selected randomly: translations $u^{(i)}$ and $w^{(i)}$ are uniformly distributed between -1 pix and 1 pix (about one tenth of the diameter of the grains), while rotations $\omega^{(i)}$ are uniformly distributed between $-6^{\circ}$ and $6^{\circ}$.

As a first test, we carried out the D-DPC optimization described in Sec. 3.1 on each grain individually. In other words, we optimized the following cost functions [compare with Eq. (16)]

$\mathrm{F}^{(i)}(\mathbf{q})=\sum_{\theta} \sum_{\underline{p}}\left(\hat{P}^{(i)}\left(\theta, \underline{p} ; \mathbf{q}^{(i)}\right)-\hat{P}^{(i)}\left(\theta, \underline{p} ; \mathbf{q}_{\text {exact }}^{(i)}\right)\right)^{2}$,

and retrieved $\mathbf{q}_{\text {D-DPC }}^{(i)}=\mathbf{q}_{\text {exact }}^{(i)}$ (up to machine accuracy) for each grain $i$.

We then tested our method on assemblies of grains. For loose assembly of grains, the rays intersect a limited number of grains. Each pixel of the sensor therefore measures information relating to a small number of grains, and we expected the D-DPC method to deliver more accurate results in this case. In order to verify this intuition, we studied three different groups of grains (subsets of the assembly shown in Fig. 3). In the first group (labeled "loose" in what follows), the six selected grains are separated by roughly two diameters (see blue squares on Fig. 3). In the second group (labeled "dense" in what follows), the six selected grains are separated by a few pixels (see red circles on Fig. 3). Finally, the tests were also carried out on all grains shown in Fig. 3. It should be noted that in all these test cases, the grains are not in contact; more realistic configurations are tested in Sec. 4. 


\begin{tabular}{l|c|c} 
& Max. rel. err. & Num. iter. \\
\hline Loose & $310^{-13}$ & 8 \\
Dense & $210^{-13}$ & 8 \\
All grains & $1310^{-13}$ & 12 \\
\hline
\end{tabular}

Table 1 Results of the tests described in Sec. 3.2.1. For all three assemblies of grains, the table reports the maximum component-wise relative error on the generalized displacement, as well as the number of iterations of the Levenberg-Marquardt algorithm.

\begin{tabular}{c|c|c|c} 
& Num. proj. & Max. rel. err. & Num. iter. \\
\hline All grains & 2 & 27 & 67 \\
All grains & 4 & 3 & 935 \\
All grains & 6 & $310^{-13}$ & 42 \\
\hline
\end{tabular}

Table 2 Results of the tests described in Sec. 3.2.2. The table reports the number of projections, the maximum component-wise relative error on the generalized displacement and the number of iterations of the Levenberg-Marquardt algorithm.

To quantify the accuracy of our method, we measure the component-wise maximal relative error of $\mathbf{q}_{\text {exact }}$ and $\mathbf{q}_{\text {D-DPC }}$. The results (averaged over five realizations of $\mathbf{q}_{\text {exact}}$ ) are reported in table 1. It is observed that the minimization is successful in all three cases. In particular, contrary to what we expected, convergence of the method is not affected by the density of the sample. This is a very desirable feature for future applications to real, experimental situations. We finally note that the number of iterations of the LevenbergMarquardt algorithm grows with the number of grains.

\subsubsection{Validation with two to six projections and large displacements}

In this section, we present tests carried out with larger displacements, defined as follows. The translations are deterministic

$u^{(i)}=\alpha x^{(i)}$,

$w^{(i)}=\beta z^{(i)}$,

with $\alpha=0.15$ and $\beta=0.1$. Rotations $\omega^{(i)}$ are sampled from a uniform distribution between $-30^{\circ}$ and $30^{\circ}$.

The error was measured as in Sec. 3.2.1 above, and the results are reported in table 2, where it is observed that the D-DPC method fails in this case with two projections $\left(\theta=22.5^{\circ}, 112.5^{\circ}\right)$. We therefore carried out two additional simulations with four $\left(\theta=22.5^{\circ}, 67.5^{\circ}, 112.5^{\circ}, 157.5^{\circ}\right)$ and $\operatorname{six}\left(\theta=22.5^{\circ}, 52.5^{\circ}, 82.5^{\circ}, 112.5^{\circ}, 142.5^{\circ}, 172.5^{\circ}\right)$ projections. With four projections, the error was still unacceptably high, while six projections led to an excellent accuracy. It is very likely that with two and four projections, the optimization algorithm converged to a local minimum. This point is discussed in the next section.

\subsection{Sensitivity to the initial guess}

The D-DPC cost function F defined by Eq. (16) is not convex. Therefore, for the Levenberg-Marquardt method to converge, the initial guess should be close enough to the global minimum. Otherwise, the D-DPC method may return a local minimum. In true, experimental conditions, the load should be applied in small increments, and the D-DPC method should be applied at each load step, using as initial guess for the current load step the converged generalized displacement at the previous load step.

In the present section, we study the sensitivity to the initial guess empirically. We consider the D-DPC cost function F corresponding to the two-dimensional, parallel projections of one grain

$$
\begin{aligned}
\mathrm{F}(u, w, \omega) & =\sum_{\theta, \underline{p}}(\hat{P}(\theta, \underline{p} ; u, w, \omega)-P(\theta, \underline{p}))^{2} \\
& =\sum_{\theta, \underline{p}}(\hat{P}(\theta, \underline{p} ; u, w, \omega)-\hat{P}(\theta, \underline{p} ; 0,0,0))^{2},
\end{aligned}
$$

where the two "experimental" projections $P(\theta, p)\left(\theta=22.5^{\circ}\right.$, $112.5^{\circ}$ ) are generated from the $2 \mathrm{D}$ image of the grain marked with a green cross on Fig. 3 (largest diameter: 37 pix). In the present example, the exact generalized displacement is $\mathbf{q}_{\text {exact }}=\mathbf{0}$, and an empirical study of this function in the neighborhood of this minimum is provided.

Our observations show that the optimization procedure is more sensitive to the initial value of the rotation $\omega$ than translations $u$ and $w$. We therefore focus in what follows on a $1 \mathrm{D}$ cross-section of the cost function: $\omega \mapsto \mathrm{F}(0,0, \omega)$, where the translations $u$ and $w$ are frozen. The resulting crosssection is plotted in Fig. 4 (left axis) for values of $\omega$ ranging from $-90^{\circ}$ to $90^{\circ}$. Clearly, the function is convex only in the neighborhood of the minimum $\omega=0$, and the initial guess of $\omega$ should be selected in this neighborhood. This is illustrated on Fig. 4 (right axis), where the symbols show the converged value of the rotation $\omega_{\mathrm{D}-\mathrm{DPC}}$, for the initial guess $\left(0,0, \omega_{\text {init }}\right)$, where $\omega_{\text {init }}$ takes the values $-90^{\circ},-45^{\circ},-22.5^{\circ}$, $-4.5^{\circ}, 4.5^{\circ}, 22.5^{\circ}, 45^{\circ}, 90^{\circ}$. The simulation is successful if the converged value of the rotation is null (up to machine accuracy). In Fig. 4, successful simulations correspond to the green squares lying on the $x$-axis. These results confirm that the initial guess must be close enough to the solution.

It should be noted that we deliberately considered extremely large rotations: true rotations usually observed in experimental conditions are much smaller [9, 10]. It is observed that succesful convergence is obtained for initial guesses of the rotation which are in error by about $30^{\circ}$. This illustrates the robustness of our method.

To close this section, we mention that the same analysis (not presented here) can be carried out on the translations. Our simulations show that convergence to the exact displacement is obtained for initial guesses of the translations which 


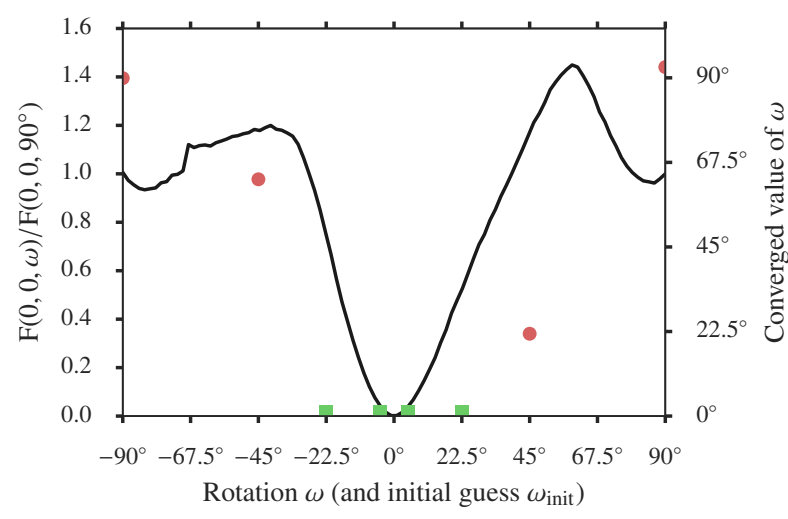

Fig. 4 Plot of the objective function F considered in Sec. 3.3 around its minimum (continuous line, left axis). $F$ is not convex for large values of $\omega$, and the initial guess ought to be close enough to the minimum, as illustrated by the symbols (right axis). See main text for a description of this plot.

are in error by several pixels. The amplitude of the convergence domain is deemed large enough for practical applications, especially if the load is applied in small increments.

3.4 Accounting for brightness and contrast evolutions of the projections

Changes of the intensity of the X-ray source and imperfections of the grey level calibration procedure can induce variations of brightness and contrast between the two series of projections. In standard DIC or V-DIC algorithms, a local brightness and contrast correction (to be optimized) is usually applied to the 3D images. Similarly, we introduce two additional optimization parameters, namely: $a$ (global scaling of the grey levels) and $b$ (global shift of the grey levels). The cost function $\mathrm{F}$ now reads

$\mathrm{F}(a, b, \mathbf{q})=\sum_{\theta} \sum_{\underline{p}}(\hat{P}(\theta, \underline{p} ; \mathbf{q})-(a P(\theta, \underline{p})+b))^{2}$,

[compare with Eq. (16)].

\subsection{Implementation of the method}

The standard Python implementation of the Levenberg-Marquardt method provided by the scipy. optimize ${ }^{1}$ was used to solve the optimization problem defined by Eq. (17).

Naturally, the bottleneck of the code is the evaluation of the cost function F defined by Eq. (16); as such, it was implemented with great care. The Cython ${ }^{2}[38,39]$ static compiler

\footnotetext{
1 https://www.scipy.org/scipylib/. Retrieved 15 December 2016.

2 http://cython. org/. Retrieved 15 December 2016.
}

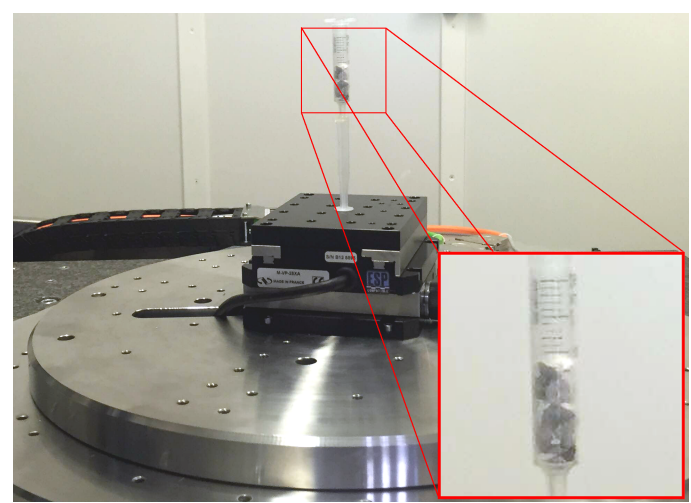

Fig. 5 The specimen considered in Sec. 4. The photograph also shows the sample stage of the tomography setup.

was used to produce a native $\mathrm{C}$-extension for Python of the projection operator $\hat{P}^{(i)}\left(\theta, \underline{p} ; \mathbf{q}^{(i)}\right)$ defined by Eq. (9). Then, observing that each term of the sum appearing in Eq. (16) can be evaluated independently, the MapReduce pattern [40] was used for the parallelization of the computation of the objective function F. This means that each available core is in charge of computing the projections of a subset of all grains.

\section{Experimental validation}

In the present section, the D-DPC methodology is applied to a simple experiment carried out on a simplified granular medium.

\subsection{Specimen}

The specimen is an assembly of 15 grains of limestone gravel from the Boulonnais quarries (mean diameter of the grains: $5 \mathrm{~mm}$, density: 2.6 ). The grains are placed in a polypropylene syringe (diameter: $10 \mathrm{~mm}$; height: $30 \mathrm{~mm}$ ), see Fig. 5. The $\mathrm{X}$-ray absorption of the container is small compared to that of gravel; it only contributes to about $10 \%$ of the sinogram (defined here loosely as the set of radiographic projections).

\subsection{X-ray microtomography and reconstruction}

X-ray microtomography experiments were performed at Laboratoire Navier with an Ultratom scanner from RX Solutions combining a Hamamatsu L10801 X-ray source $(230 \mathrm{kV}$, $200 \mathrm{~W}$, smallest spot size: $5 \mu \mathrm{m}$ ) and a Paxscan Varian $2520 \mathrm{~V}$ flat-panel imager $\left(1920 \times 1560 \mathrm{pix}^{2}\right.$, pixel size $\left.127 \mu \mathrm{m}\right)$.

All scans were performed at $100 \mathrm{kV}$ and $500 \mu \mathrm{A}$, with a frame rate of 2 images per second. Each projection is the result of averaging 40 images. Owing to the rather large effective exposure time $(20 \mathrm{~s})$, the signal-to-noise ratio reaches approximately 50 (see below), which allowed us to neglect 


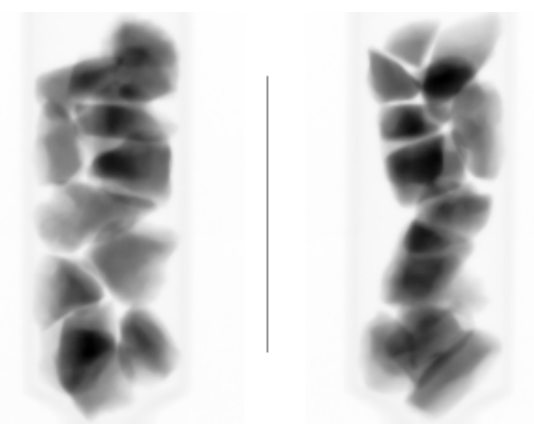

Fig. 6 Two orthogonal projections of the specimen used for validating the D-DPC method (see Sec. 4).

noise-induced errors in the evaluation of the D-DPC method presented below. Further studies (to be reported elsewhere) indeed confirm that noise-induced errors are dominated by discretization and modelling errors.

According to the geometry of the tomography setup, the voxel size was estimated to $0.112 \mathrm{~mm} \cdot$ vox $^{-1}$. Fig. 6 shows two orthogonal projections of the sample.

Three full scans (352 radiographs spanning the full $360^{\circ}$ ) were then performed. For scans 1 and 2, the position of the sample was unchanged (reference configuration), while for scan 3 , a $3.5 \mathrm{~mm}( \pm 0.1 \mu \mathrm{m}$, accuracy of moving stage $)$ translation in the $O x z$ plane (see Fig. 2) was applied to the sample.

The D-DPC method is based on an algebraic projection operator. Consistency then requires that the initial configuration be reconstructed by means of the same projection operator (as was also observed by Leclerc and coauthors [24]). This of course precludes the use of efficient reconstruction techniques such as the Filtered Back-Projection. Instead, we implemented the Simultaneous Algebraic Reconstruction Technique (SART), parallelism being provided by the Portable Extensible Toolkit for Scientific Computation (PETSc) [41, 42]. Our implementation was then applied to a $180 \times 280$ pix $^{2}$ region of interest of the detector (leading to a $180 \times 280 \times 180$ vox $^{3}$ reconstructed volume) .

In order to set up a stopping criterion for the SART iterations, we first defined the relative residual error $\epsilon$

$\epsilon=\frac{\|b-A x\|_{2}}{\|b\|_{2}}$

where $A$ denotes the projection operator, $b$ denotes the sinogram, and $x$ is the unknown reconstruction. For all three scans, the iterations were stopped when no significant reduction of the error $\epsilon$ was observed.

In all cases, this led to $\epsilon \simeq 3 \%$, which is consistent with a stopping criterion based on the discrepancy principle [43]. Indeed, it should be observed that the specimen was not moved between scans 1 and 2 . In other words, the projections from these two scans are essentially identical, up to noise. Their difference therefore gives a reliable estimate of the

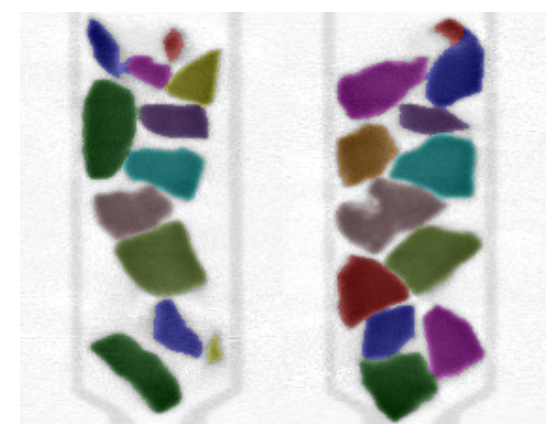

Fig. 7 Two orthogonal cross-sections through the segmented, reconstructed volume.

signal-to-noise (SNR) ratio, which was computed as follows

$\mathrm{SNR}=\frac{1}{2} \frac{\left\|b_{1}+b_{2}\right\|_{2}}{\left\|b_{2}-b_{1}\right\|_{2}}$

where $b_{1}, b_{2}$ denote the sinograms from the first and second scans, respectively. We found that $1 / \mathrm{SNR} \simeq 2 \%$. The residual error at the end of the iterative reconstruction was therefore comparable to the amplitude of the noise, which validates our stopping criterion.

\subsection{Segmentation of the reconstructed volume}

Segmentation of the reconstructed volume (reference configuration) is a crucial step of our method, since the geometry of each grain is required for the determination of their displacements. We applied the watershed algorithm [44, 45] to the distance transform of the thresholded image. To avoid over-segmentation, regional minima whose depth was less than a specified threshold were suppressed by means of the $\mathrm{H}$ minima transform [46]. Fig. 7 shows the resulting segmented image.

\subsection{Application of the D-DPC method}

The segmented 3D reconstruction resulting from Scan 1 was used as a reference configuration for the generation of trial projections within the framework of the D-DPC method, which was then applied to Scans 2 and 3, successively.

Application to Scan 2 The specimen is untransformed; therefore, our method should converge to null displacements for all grains. Deviations from this expected result provide an estimate of the accuracy of the D-DPC method.

Application to Scan 3 The specimen was subjected to a $3.5 \mathrm{~mm}$ (31.25 vox) translation in the $O x z$ plane; therefore, the D-DPC method should converge to the same rigid body motion for all grains. The expected value of this rigid body 


\begin{tabular}{c|c|c|c} 
& $X$ & $Y$ & $Z$ \\
\hline$\underline{u}[\mathrm{~mm}]$ & $0.00(0.04)$ & $0.00(0.02)$ & $0.01(0.03)$ \\
$\underline{u}[\mathrm{vox}]$ & $0.0(0.3)$ & $0.0(0.2)$ & $0.1(0.3)$ \\
$\underline{\omega}\left[^{\circ}\right]$ & $-0.2(0.7)$ & $-0.2(0.9)$ & $0.1(0.8)$ \\
\hline
\end{tabular}

Table 3 Application of the D-DPC method to Scan 2 (see Sec. 4.4). The components of the translation $\underline{u}$ and the rotation $\underline{\omega}$ are averaged over all grains. Figures in parentheses are standard deviations over the grains.

\begin{tabular}{c|c|c|c} 
& $X$ & $Y$ & $Z$ \\
\hline$\underline{u}[\mathrm{~mm}]$ & $2.76(0.03)$ & $0.01(0.04)$ & $-2.12(0.04)$ \\
$\underline{u}[\mathrm{vox}]$ & $24.7(0.3)$ & $0.1(0.4)$ & $-18.9(0.4)$ \\
$\underline{\omega}\left[^{\circ}\right]$ & $-0.2(1.5)$ & $-0.8(1.9)$ & $0.3(0.8)$ \\
\hline
\end{tabular}

Table 4 Application of the D-DPC method to Scan 3 (see Sec. 4.4). The components of the translation $\underline{u}$ and the rotation $\underline{\omega}$ are averaged over all grains. Figures in parentheses are standard deviations over the grains.

motion was estimated by means of the standard Volumetric Digital Image Correlation technique (VDIC) [17], leading to

$$
\begin{aligned}
\mathbf{q}_{\mathrm{VDIC}} & =(2.78 \mathrm{~mm}, 0.00 \mathrm{~mm},-2.12 \mathrm{~mm}), \\
& =(24.8 \text { vox, } 0.0 \text { vox },-18.9 \text { vox })
\end{aligned}
$$

$\left\|\mathbf{q}_{\text {vDIC }}\right\|=3.50 \mathrm{~mm}$.

Again, deviations from this expected result provide an estimate of the accuracy of the D-DPC method.

In both cases, only 4 projections $\left(0^{\circ}, 45^{\circ}, 90^{\circ}, 135^{\circ}\right)$ were used to estimate the rigid body motion of each grain. The results are presented in Tables 4.4 (Scan 2) and 4.4 (Scan 3 ). The measured translations and rotations are averaged over all grains, while the corresponding standard deviation is used as a measure of the method accuracy. For both scans, we found that the accuracy was about $0.01 \mathrm{~mm}$ ( 0.1 vox ) for translations and $1^{\circ}$ for rotations.

It is again emphasized that only 4 projections were required to achieve the reported accuracy, although the initial guess passed to the optimization algorithm provided purposedly a very poor estimate of the true displacements. Indeed, the reference configuration was used as initial guess $\left(\mathbf{q}_{\text {init }}=0\right)$. If the initial guess is chosen within 1 vox (translations) and $6^{\circ}$ (rotations) of the true displacements, then the accuracy achieved with only 2 projections (the bare minimum) was similar for translations, and only slightly degraded for rotations.

To close this section, we note that Leclerc and coauthors [24] report similar accuracies when using very few projections.

\section{Conclusion}

In this paper, we have proposed a new method based on $\mathrm{X}$ ray microtomography to capture the movements of grains within granular materials. The most salient feature of this method, wich we called D-DPC (Discrete Digital Projection Correlation), is that it does not require a $3 \mathrm{D}$ reconstruction of the specimen in its current (deformed) state (it does require a reconstruction of the specimen in its initial state). Our tests show that as few as two projections suffice to deliver a satisfactory estimate of the displacements. This results in a dramatic reduction of the acquisition time, therefore allowing for time-dependent phenomena (such as creep) to be studied.

The D-DPC method is formulated as an inverse problem, which is fully stated in the present paper. Both synthetic and real-life test cases confirm the value of the method, which is accurate to about 0.1 vox (translations) and $1^{\circ}$ (rotations) in standard laboratory experimental conditions. Although more conventional correlation techniques can achieve more accurate measurements, we believe that this is largely compensated by the significant gain in acquisition time that our method offers. Besides, we are currently investigating the potential sources of errors (noise, fluctuations of the beam, ...) as well as several ways to improve the accuracy of DDPC, such as refining the projection model, accouting for geometric imperfections of the tomography setup, ...

Acknowledgements This work has benefited from a French government grant managed by ANR within the frame of the national program Investments for the Future ANR-11-LABX-022-01.

The authors would like to thank Matthieu Vandamme for fruitful discussions.

\section{References}

1. Wan R, Guo P (1998) A simple constitutive model for granular soils: Modified stress-dilatancy approach. Computers and Geotechnics 22(2):109-133, DOI 10.1016/ S0266-352X(98)00004-4

2. Sanzeni A, Whittle A, Germaine J, Colleselli F (2012) Compression and creep of venice lagoon sands. Journal of Geotechnical and Geoenvironmental Engineering 138(10):1266-1276, DOI 10.1061/(ASCE)GT. 1943-5606.0000696

3. Blanc B, Géminard JC (2013) Intrinsic creep of a granular column subjected to temperature changes. Physical Review E 88:022,201, DOI 10.1103/PhysRevE.88. 022201

4. Karimpour H, Lade P (2013) Creep behavior in Virginia Beach sand. Canadian Geotechnical Journal 50(11):1159-1178, DOI 10.1139/cgj-2012-0467w

5. Kak A, Slaney M (2001) Principles of computerized tomographic imaging. Society for Industrial and Applied Mathematics

6. Hsieh J (2009) Computed tomography: principles, design, artifacts, and recent advances, 2 nd edn. Wiley 
7. Desrues J (2004) Tracking strain localization in geomaterials using computerized tomography. In: Otani J, Obara Y (eds) Proceedings of the 1st international workshop on X-ray computerized tomography for geomaterials, Kumamoto, Japan, Balkema, pp 15-41

8. Desrues J, Chambon R, Mokni M, Mazerolle F (1996) Void ratio evolution inside shear bands in triaxial sand specimens studied by computed tomography. Géotechnique 46(3):529-546, DOI 10.1680/geot.1996. 46.3.529

9. Hall S, Bornert M, Desrues J, Pannier Y, Lenoir N, Viggiani G, Bésuelle P (2010) Discrete and continuum analysis of localised deformation in sand using X-ray $\mu \mathrm{CT}$ and volumetric digital image correlation. Géotechnique 60(5):315-322, DOI 10.1680/geot.2010.60.5.315

10. Andò E, Hall S, Viggiani G, Desrues J, Bésuelle P (2012) Grain-scale experimental investigation of localised deformation in sand: a discrete particle tracking approach. Acta Geotechnica 7(1):1-13, DOI 10.1007/ s11440-011-0151-6

11. Radjai F, Dubois F (eds) (2011) Discrete-element Modeling of Granular Materials. Wiley-ISTE

12. Cundall P, Strack O (1979) A discrete numerical model for granular assemblies. Geotechnique 29(1):47-65, DOI 10.1680/geot.1979.29.1.47

13. Thornton C (2010) Quasi-static simulations of compact polydisperse particle systems. Particuology 8(2):119 126, DOI 10.1016/j.partic.2009.07.007

14. Bay B, Smith T, Fyhrie D, Saad M (1999) Digital volume correlation: Three-dimensional strain mapping using Xray tomography. Experimental Mechanics 39(3):217226, DOI 10.1007/BF02323555

15. Bornert M, Chaix JM, Doumalin P, Dupré JC, Fournel T, Jeulin D, Maire E, Moreaud M, Moulinec H (2004) Mesure tridimensionnelle de champs cinématiques par imagerie volumique pour l'analyse des matériaux et des structures. Instrumentation, Mesure, Métrologie 4(34):43-88

16. Germaneau A, Doumalin P, Dupré JC (2008) Comparison between X-ray micro-computed tomography and optical scanning tomography for full $3 \mathrm{~d}$ strain measurement by digital volume correlation. NDT \& E International 41(6):407-415, DOI 10.1016/j.ndteint.2008.04.001

17. Lenoir N, Bornert M, Desrues J, Bésuelle P, Viggiani G (2007) Volumetric digital image correlation applied to Xray microtomography images from triaxial compression tests on argillaceous rock. Strain 43(3):193-205, DOI 10.1111/j.1475-1305.2007.00348.x

18. Roux S, Hild F, Viot P, Bernard D (2008) Threedimensional image correlation from X-ray computed tomography of solid foam. Composites Part A: Applied Science and Manufacturing 39(8):1253-1265, DOI 10.1016/j.compositesa.2007.11.011
19. Desrues J, Andò E (2015) Strain localisation in granular media. Comptes Rendus Physique 16(1):26-36, DOI 10.1016/j.crhy.2015.01.001

20. Lhuissier P, Scheel M, Di Michiel M, Boller E, Adrien J, Maire E, Salvo L, Blandin JJ, Suery M (2012) Ultra fast tomography: New developments for $4 \mathrm{~d}$ studies in material science. In: De Graef M, Friis Poulsen H, Lewis A, Simmons J, Spanos G (eds) 1st International Conference on 3D Materials Science, John Wiley \& Sons, Inc., pp 203-208, DOI 10.1002/9781118686768.ch31

21. Limodin N, Salvo L, Suéry M, DiMichiel M (2007) In situ investigation by X-ray tomography of the overall and local microstructural changes occurring during partial remelting of an Al-15.8 wt.\% Cu alloy. Acta Materialia 55(9):3177-3191, DOI 10.1016/j.actamat.2007.01.027

22. Mader K, Marone F, Hintermüller C, Mikuljan G, Isenegger A, Stampanoni M (2011) High-throughput fullautomatic synchrotron-based tomographic microscopy. Journal of Synchrotron Radiation 18(2):117-124, DOI 10.1107/S0909049510047370

23. Leclerc H, Roux S, Hild F (2013) X-CT Digital Volume Correlation without Reconstruction. In: Cnudde V, Bernard D (eds) 1st International Conference on Tomography of Materials and Structures (ICTMS 2013), Ghent, Belgium, pp 265-268

24. Leclerc H, Roux S, Hild F (2015) Projection savings in CT-based digital volume correlation. Experimental Mechanics 55(1):275-287, DOI 10.1007/ s11340-014-9871-5

25. Taillandier-Thomas T, Leclerc H, Roux S, Hild F (2015) Projection-based digital volume correlation: application to crack propagation. In: Long B, Francus P (eds) 2nd International Conference on Tomography of Materials and Structures (ICTMS 2015), Québec, Canada, p 124

26. Taillandier-Thomas T, Roux S, Hild F (2016) Soft route to 4d tomography. Physical Review Letters 117:025,501, DOI 10.1103/PhysRevLett.117.025501

27. Sidky E, Kao C, Pan X (2006) Accurate image reconstruction from few-views and limited-angle data in divergent-beam CT. Journal of X-ray science and technology 14(2):119-139

28. Ketcham R, Carlson W (2001) Acquisition, optimization and interpretation of X-ray computed tomographic imagery: applications to the geosciences. Computers \& Geosciences 27(4):381-400, DOI 10.1016/ S0098-3004(00)00116-3

29. Maire E, Buffire JY, Salvo L, Blandin JJ, Ludwig W, Ltang JM (2001) On the application of $\mathrm{x}$-ray microtomography in the field of materials science. Advanced Engineering Materials 3(8):539546, DOI 10.1002/1527-2648(200108)3:8<539:: AID-ADEM539>3.0.CO;2-6 
30. Boas FE, Fleischmann D (2012) CT artifacts: causes and reduction techniques. Imaging in Medicine 4(2):229 240, DOI 10.2217/iim.12.13

31. Rodrigues O (1840) Des lois géométriques qui régissent les déplacements d'un système solide dans l'espace, et de la variation des coordonnées provenant de ces déplacements considérés indépendamment des causes qui peuvent les produire. Journal de Mathématiques Pures et Appliquées 5:380-440

32. Argyris J (1982) An excursion into large rotations. Computer Methods in Applied Mechanics and Engineering 32(1-3):85-155, DOI 10.1016/0045-7825(82)90069-X

33. Cardona A, Geradin M (1988) A beam finite element non-linear theory with finite rotations. International Journal for Numerical Methods in Engineering 26(11):24032438, DOI 10.1002/nme.1620261105

34. Ibrahimbegović A, Frey F, Kožar I (1995) Computational aspects of vector-like parametrization of threedimensional finite rotations. International Journal for Numerical Methods in Engineering 38(21):3653-3673, DOI 10.1002/nme.1620382107

35. Siddon R (1985) Fast calculation of the exact radiological path for a three-dimensional CT array. Medical Physics 12(2):252-255, DOI 10.1118/1.595715

36. Jacobs F, Sundermann E, De Suiter B, Christiaens M, Lemahieu I (1998) A fast algorithm to calculate the exact radiological path through a pixel or voxel space. Journal of computing and information technology 6(1):89-94

37. Shepp L, Vardi Y (1982) Maximum likelihood reconstruction in positron emission tomography. IEEE Transactions on Medical Imaging 1(2)

38. Behnel S, Bradshaw R, Citro C, Dalcin L, Seljebotn D, Smith K (2011) Cython: The best of both worlds. Computing in Science Engineering 13(2):31 -39, DOI 10.1109/MCSE.2010.118

39. Smith KW (2015) Cython - A Guide for Python Programmers. O’Reilly Media, Inc., 1005 Gravenstein Highway North, Sebastopol, CA 95472

40. McCool M, Robison AD, Reinders J (2012) Structured Parallel Programming. Morgan Kaufmann, 225 Wyman Street, Waltham, MA 02451, USA

41. Balay S, Gropp WD, McInnes LC, Smith BF (1997) Efficient management of parallelism in object oriented numerical software libraries. In: Arge E, Bruaset AM, Langtangen HP (eds) Modern Software Tools in Scientific Computing, Birkhäuser Press, pp 163-202

42. Balay S, Abhyankar S, Adams MF, Brown J, Brune P, Buschelman K, Dalcin L, Eijkhout V, Gropp WD, Kaushik D, Knepley MG, McInnes LC, Rupp K, Smith BF, Zampini S, Zhang H (2015) PETSc users manual. Tech. Rep. ANL-95/11 - Revision 3.6, Argonne National Laboratory, URL http: //www . mcs . anl . gov/petsc, accessed 08-04-2016
43. Morozov VA (1984) Methods for Solving Incorrectly Posed Problems. Springer-Verlag

44. Beucher S (1992) The watershed transformation applied to image segmentation. In: Scanning Microscopy International, Cambridge, UK, vol suppl. 6, pp 299-314

45. Beucher S, Lantuéjoul C (1979) Use of watersheds in contour detection. In: International Workshop on Image Processing: Real-time Edge and Motion Detection/Estimation, Rennes, France

46. Soille P (2004) Morphological Image Analysis: Principles and Applications, 2nd edn. Springer-Verlag, Berlin Heidelberg 